\title{
Who dunnit? Cross-linguistic differences in eye-witness memory
}

\author{
Caitlin M. Fausey $\cdot$ Lera Boroditsky
}

Published online: 17 November 2010

(C) Psychonomic Society, Inc. 2010

\begin{abstract}
Does eye-witness memory differ depending on the language one speaks? We examined English and Spanish speakers' descriptions of intentional and accidental events, and their memory for the agents of these events. English and Spanish speakers described intentional events similarly, using mostly agentive language (e.g., "She broke the vase"). However, when it came to accidental events English speakers used more agentive language than did Spanish speakers. Results from a non-linguistic memory task mirrored the patterns in language. English and Spanish speakers remembered the agents of intentional events equally well. However, English speakers remembered the agents of accidental events better than did Spanish speakers. Together these findings demonstrate that there are cross-linguistic differences in event descriptions that have important consequences for eye-witness memory.
\end{abstract}

Keywords Language $\cdot$ Language and thought $\cdot$ Eye-witness memory $\cdot$ Events $\cdot$ Causality $\cdot$ Agents $\cdot$ Accident

Are there cross-linguistic differences in eye-witness memory? Can patterns in our linguistic environment influence what we remember about the events we witness? In this paper we identify a cross-linguistic difference in how English and Spanish speakers describe the same events, and find that there is a corresponding cross-linguistic difference in eye-witness memory.

\footnotetext{
C. M. Fausey $(\bowtie)$

Department of Psychological and Brain Sciences, Indiana University,

1101 E. 10th Street,

Bloomington, IN 47405, USA

e-mail: cfausey@indiana.edu

L. Boroditsky

Department of Psychology, Stanford University,

Jordan Hall, 450 Serra Mall,

Stanford, CA 94305 2130, USA
}

You see someone accidentally brush against a flower vase and the vase ends up in pieces on the floor. When asked about what happened, you might say, "She broke the vase." In English, agentive descriptions like this are typical and appropriate even for clearly accidental events. By contrast, non-agentive language often sounds evasive (e.g., Reagan's famous "mistakes were made" in the 1987 State of the Union Address). Linguistic analyses suggest that in other languages, non-agentive expressions are more frequent and used to distinguish accidental from intentional actions (Dorfman, 2004; Filipovic, 2007; Maldonado, 1992; Martinez, 2000; Slobin \& Bocaz, 1988). For example, in Spanish nonagentive expressions with the clitic se are often used to describe accidents (e.g., "Se rompió el florero", translating roughly as "The vase broke itself").

\footnotetext{
${ }^{1}$ The clitic se serves a variety of functions in Spanish. Among other uses, Spanish se expressions may be considered reflexive (Se peinó; He combed his hair), impersonal (Aqui se habla español; Spanish is spoken here), passive (Se vendieron los coches; The cars were sold), reciprocal (Se abrazaron; They hugged each other), and a marker of accidentality (La taza se rompió; The cup broke). Some semantically oriented analyses have suggested that different se usages are connected in a network of related meanings. On one proposal, se expressions encourage event perceptions in which the causal initiator is schematic and underspecified, and the results of events are highlighted (e.g., Maldonado, 1992). Some syntactically oriented analyses have proposed that se expressions are derived from transitive expressions, "losing" an argument in the process (e.g., Grimshaw, 1982; see also Maldonado, 1992 for a thorough discussion of several approaches). For example, the se expression "El florero se rompió" would be derived from the transitive expression "Jon rompió el florero," deleting the argument "Jon." Though terminology and conclusions about the syntax and semantics of Spanish se vary across theoretical approaches, the main thrust is that many uses of Spanish se appear to highlight outcomes more than causes. Here, we offer a usage-based approach to examining Spanish se and test whether its use as an "accidentality marker" impacts memory for causal events.
} 
Previous work in English has shown that alternations between agentive and non-agentive descriptions can have important consequences for how people reason about events (Fausey \& Boroditsky, 2010). For example, English speakers who read a report about Justin Timberlake and Janet Jackson's wardrobe malfunction containing the agentive expression "tore the bodice" not only blamed Timberlake more, but also levied 53\% more in fines than those who read the non-agentive "the bodice tore." Further, this linguistic framing had a big effect on blame and punishment even when people watched a video of the event and were able to witness the tearing with their own eyes.

In this paper, we investigate whether agentivity in event descriptions also affects eye-witness memory. If events would normally be described less agentively in your linguistic community, would you be less likely to pay attention to and remember the agents of those events than if they were normally described more agentively? Previous work has examined the role of linguistic framing in eyewitness memory within a language by presenting participants with different descriptions of the same event, for example varying the vividness of verbs, and measuring effects on memory (e.g., Gentner \& Loftus, 1979; Loftus \& Palmer, 1974).

The studies in this paper extend this work to the crosslinguistic domain. In a typical within-language framing study (e.g., Gentner \& Loftus, 1979; Loftus \& Palmer, 1974), the experimenter provides participants with a onetime linguistic framing of a single event and tests if the description influences how people remember that specific event. This type of study demonstrates that explicit linguistic framing can have immediate and specific effects on memory. Cross-linguistic differences in event descriptions allow us to extend this logic from explicit one-time framing to examine potential effects of habitual framing. Without the need for an explicit frame provided by the experimenter, cross-linguistic research allows us to test how habitual patterns in natural language may guide memory.

In this paper, instead of giving participants different descriptions of the same event, we show the same silent events to participants from two different linguistic communities. We ask whether habitual patterns of linguistic framing in the two different linguistic communities will lead the two groups of participants to naturally pay attention to, encode, and remember different aspects of the same event. That is, are speakers of different languages habitually operating in different linguistic framing conditions as a function of how events are normally described in their linguistic community? Can effects of these differences be seen even in the absence of explicit linguistic framing during the task?

Much previous cross-linguistic work on the role of language in event cognition has focused on cross-linguistic differences in encoding the manner and path of motion (e.g.,
Billman \& Krych, 1998; Finkbeiner, Nicol, Greth, \& Nakamura, 2002; Gennari, Sloman, Malt, \& Fitch, 2002; Oh, 2003; Papafragou, Hulbert, \& Trueswell, 2008; Papafragou, Massey, \& Gleitman, 2002; Slobin, 2003; Trueswell \& Papafragou, 2010). Many of these studies have found cross-linguistic differences in how people encode and reason about motion events (e.g., Finkbeiner et al., 2002; Gennari et al., 2002; Oh, 2003; Papafragou et al., 2008; Slobin, 2003; Trueswell \& Papafragou, 2010), though some find such differences only when people are explicitly instructed to describe the events during the task (e.g., Gennari et al., 2002; Papafragou et al., 2008). Observing a cross-linguistic difference on a test of cognitive performance even when people are not required to use language in the task has become the gold standard for establishing basic cross-linguistic differences in cognition. In this paper, we specifically test for cross-linguistic differences in memory for causal events in a task where participants are not asked to describe the events at any time before or during the memory task.

In Study 1 we establish that there is indeed a difference between Spanish and English speakers' descriptions of the same causal events, and in Study 2 we test whether these differences in language have consequences for people's eye-witness memory. We hypothesized that more agentive language in one's linguistic environment would lead to better memory for the agents of events.

\section{Study 1}

\section{Method}

Participants Sixty-eight English speakers (mean age = 31.49 years) and 29 Spanish speakers (mean age = 28.69 years) participated. Participants were monolinguals who completed the study via Amazon's Mechanical Turk service (https://www.mturk.com/mturk/welcome). All participants reported that their native language, and over $80 \%$ of their current daily language use (mean $=99.98 \%$ ), is the target language and that they did not start learning any other language until after age $12 .^{2}$

Materials Participants read instructions in either English or Spanish. Instructions in the two languages were developed simultaneously and verified by an independent SpanishEnglish bilingual.

\footnotetext{
${ }^{2}$ A total of 152 people completed the study. Data from participants who provided ungrammatical or infelicitous descriptions $(N=7)$ or failed to meet the language background criteria $(N=48)$ were not analyzed.
} 
Table 1 Causal event stimuli

\begin{tabular}{lll}
\hline Action & Intentional & Accidental \\
\hline $\begin{array}{ll}\text { Crumple can } \\
\text { (Crumple cup) }\end{array}$ & $\begin{array}{l}\text { Crumples can on floor by stepping on it } \\
\text { Knock box }\end{array}$ & $\begin{array}{l}\text { Turns to walk and crumples can on floor by stepping on it } \\
\text { (Reaches to move cup, grabs too hard and crumples it) }\end{array}$ \\
Knock cups & Faces cup tower, swipes, knocks down tower & While gesturing, knocks box off table, reaches to grab it \\
Close book & Reading book, then turns head and closes book & Faces cup tower, reaches for a cup, knocks down tower \\
Rip paper & Sits at table, rips page from notebook & Reading book, then turns to look at something and closes book \\
Turn off light & Using hand, hits switch and turns off light & Sits at table, turns page in notebook and rips it \\
Spill water & Spills water by an outdoor plant & By leaning against wall, hits switch and turns off light \\
Crack egg & Takes egg from carton, cracks it against bowl & While watering outdoor plant, spills water \\
Close drawer & Faces table with open drawer, closes it with knee & As picking up egg from carton, cracks it against bowl \\
Pop balloon & Pops balloon using tack & Turning away from table with open drawer, closes it with knee \\
Open umbrella & Stands with closed umbrella, then opens it & Reaches to put tack in container, pops balloon during reach \\
Open door & By turning doorknob, opens door & Stands with closed umbrella, jumps back as opens it \\
Drop keys & Drops keys onto table & By leaning too hard against door, opens it and stumbles \\
Break pencil & Sits at table, breaks pencil in half & Attempts to put keys on table, but drops them on floor \\
Stick sticker & Places nametag sticker on shirt & Sits at table, breaks pencil in half while writing \\
Release balloon & Sits among balloons, releases one that is untied & Flops arm onto table without looking, then sticker is on arm \\
\hline
\end{tabular}

Videos of intentional and accidental versions of 16 unique events were used (Table 1). In all events, a man physically interacted with an object. The man's reaction differed between the intentional and accidental versions of the event. For example, in the intentional version of the pencil-breaking event, a man who was seated at a desk picked up a pencil, deliberately broke it in half, and looked satisfied. In the accidental version of this event, a man was writing, and while writing the pencil broke in half. In this case, the man showed a startle response and threw his hands up in surprise. Thus, the accidental events were characterized by a "whoops!" reaction such as a startle response, surprised facial expressions, and/or surprised hand gestures. Videos of eight events (both intentional and accidental versions) featured an actor in a blue shirt, and videos of another eight events (both intentional and accidental versions) featured a different actor in a yellow shirt.

Procedure Participants watched 16 videos and were asked to provide a linguistic description for each video. In each description trial, participants viewed a video and then answered the question "What happened?" (“¿Qué pasó?”). Each video showed a different event; half featured the actor in blue and half the actor in yellow; half were intentional actions and half accidental. Whether an event was presented in its intentional or accidental version was counterbalanced across participants. Videos were presented in one of two pseudo-random orders that ensured that no more than three videos of the same agent or the same intention appeared in a row.

\section{Results}

Descriptions were coded as agentive if the sentence described the change-of-state event using a transitive expression. A canonical agentive description would be "He popped the balloon." Descriptions were coded as nonagentive if the change-of-state event was described intransitively. A canonical non-agentive description would be "The balloon popped." Some non-agentive descriptions took the form "Someone was doing $X$ and then $Y$ happened," in which the agent was linguistically separated from a change-of-state event that was described intransitively. Most (84.21\%) of the Spanish non-agentive sentences were marked by the clitic $s e .^{3}$ Across all participants, $7.54 \%$ of descriptions did not describe the event and were excluded from analyses. All descriptions were coded by the first author and an independent rater, with high point-to-point reliability (97.15\% English, 96.12\% Spanish). Disagreements were resolved upon discussion. ${ }^{4}$

Results are shown in Figs. 1 and 2a. Intentional events were described equally agentively by both English and Spanish speakers (English $M=95.50, S E=.95$; Spanish

\footnotetext{
${ }^{3}$ Spanish verbs may be used intransitively without se and were sometimes used this way in our data [e.g., caer (to fall), salir (to leave), reventar (to burst)].

${ }^{4}$ Three participants (1 English, 2 Spanish) were excluded from subsequent analyses because over a third of their descriptions did not describe the event.
} 
(a)

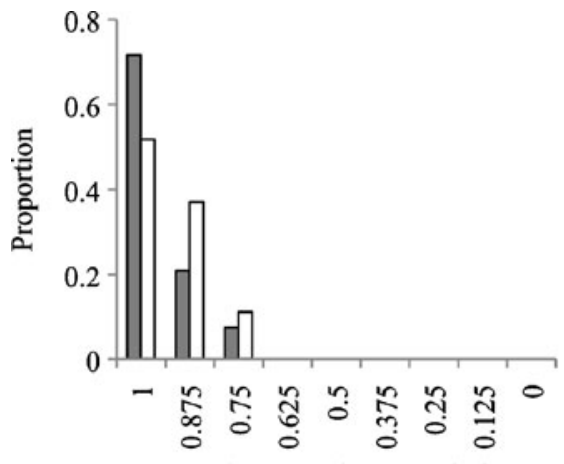

Proportion Agentive Descriptions
English

Spanish

(b)

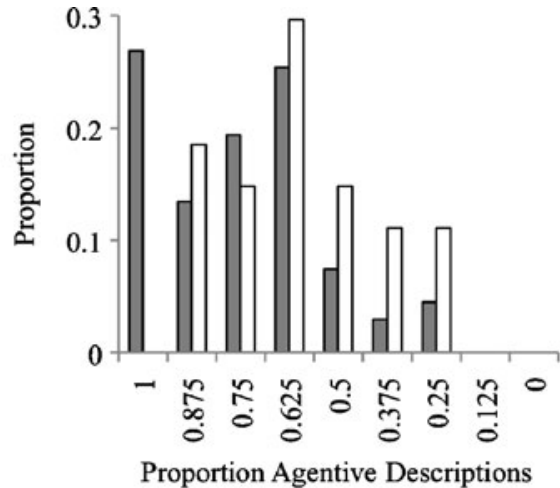

(c)

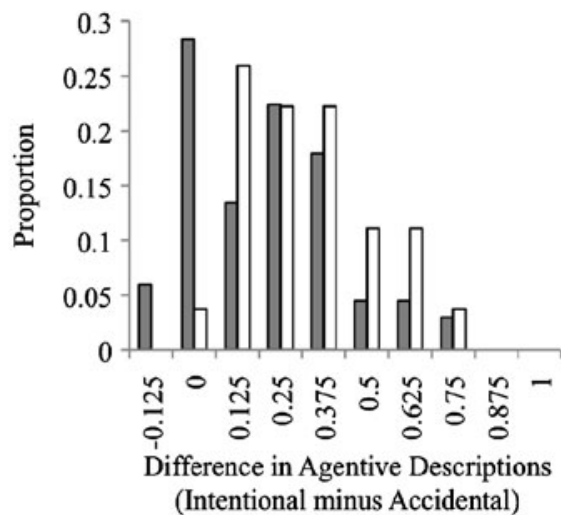

Fig. 1 Distributions of causal event descriptions in English and Spanish: (a) Intentional, (b) Accidental, (c) Difference (Intentional minus Accidental). Histograms (with proportion of the sample on the $y$-axis) of the proportion of agentive language use in each language community are plotted

20.85 years) participated. Participants were selected to be under 25 years old to ensure a homogenous sample for memory performance. All participants were monolingual, by the same criteria used in Study 1 (mean target language use $=98.84 \%$ ). None of the participants had taken part in Study 1.

General design Participants read instructions in either English or Spanish. All participants completed two tasks, first the Object-orientation memory task and then the Agent memory task. The first task was designed to be a measure of memory performance that was not predicted to vary across language communities. The second task was designed to test for differences in non-linguistic eyewitness memory (memory for the agents of events) between English and Spanish speakers. The two tasks were nonlinguistic measures of memory - participants never described any of the images or events during these two tasks, nor were they provided with any linguistic descriptions.

\section{Object-orientation memory}

During encoding, participants saw pictures of 15 objects presented on a computer screen one at a time for $2 \mathrm{~s}$ each (images courtesy of Michael J. Tarr, Brown University, http://www.tarrlab.org/). Each object appeared in one of three possible orientations, counterbalanced across participants. Participants were instructed to pay attention to the images and were told that their memory would be tested.

After the encoding phase, participants were given a brief distracter task (counting the number of white squares on a $\overline{{ }^{5} \text { All conclusions }}$ are also supported by non-parametric analyses (Mann-Whitney U and Wilcoxon signed ranks). 
Fig. 2 Describing and remembering agents in English and Spanish: (a) Causal event descriptions, with the mean proportion of agentive descriptions plotted on the y-axis. (b) Causal agent memory, with mean proportion correct plotted on the y-axis. Error bars are \pm 1 SEM (a)

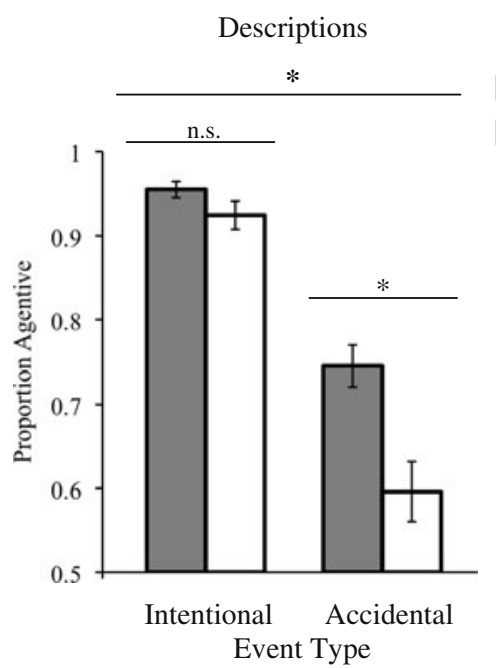

(b)

Memory

English

Spanish

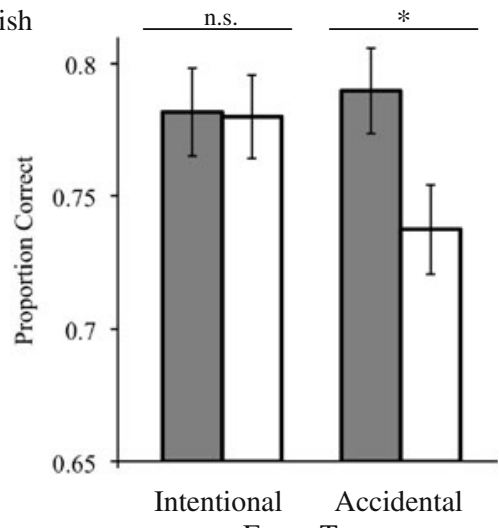

grid of black and white squares), followed by the memory test. For the memory test, participants were shown the three possible orientations of each object and asked to indicate which one they had seen previously.

\section{Agent memory}

Video materials For the encoding phase, the same videos were used as in Study 1. ${ }^{6}$ For the test phase, we used an additional set of videos showing all the same events but with actions performed by a new, third actor. The same silent videos served as stimuli for both English and Spanish speakers.

Encoding During the encoding phase, participants viewed 16 videos, following the same counterbalancing scheme as Study 1. Each video showed a different event (half featured the actor in blue and half the actor in yellow; half were intentional actions and half accidental). Participants were instructed to pay attention to the videos and were told that their memory would be tested, but were not given any further clues. After viewing all 16 videos (once each, with a one second pause between videos), participants were instructed to count to 10 as a brief distracter task.

Test Test trials consisted of a probe video followed by still photos of the two agents from the encoding phase. In the probe videos, a third actor appeared as the agent of the

\footnotetext{
${ }^{6}$ One of the 16 events - a crumpling scene - was filmed in two versions and due to a presentation error roughly half of participants in Study 2 saw a crumple video using a plastic cup and others saw a crumple video using a soda can (cup: $N=46$ Spanish, $N=65$ English). No reliable differences between these two groups were observed, and so data were pooled. All participants in Study 1 viewed the soda can stimulus.
}

same events participants saw during encoding. For example, if a participant had seen the "accidental balloon popping" event during encoding, they would see this same event acted by the new agent in the test phase. After each probe video, participants were asked, "Who did it the first time?" ("¿Quién lo hizo la primera vez?”) and responded by clicking on either the blue-shirt man or the yellow-shirt man. Participants were tested only on the events they had seen during encoding, presented in a different pseudorandom order from the encoding phase, and received no feedback.

\section{Results}

Twelve participants were excluded from analyses for one of the following reasons: (1) chance performance on the object-orientation memory task (1 English, 3 Spanish) or (2) a $\mathrm{Z}$-score greater than $|2|$ (relative to language group) on the Memory Difference Score (Intentional Memory minus Accidental Memory) (2 English, 6 Spanish). The Memory Difference Score was the basis for the analysis of interest in this study, and we wanted to be sure that outliers did not drive any observed differences. ${ }^{7}$

Results are shown in Fig. 2b. Intentional agents were remembered well by both English $(M=78.18, S E=1.66)$ and Spanish $(M=78.00, S E=1.57)$ speakers, $t(208)=.08$, n.s. However, as predicted, accidental agents were better remembered by English speakers $(M=78.98, S E=1.61)$ than by Spanish speakers $(M=73.75, S E=1.67), t(208)=$ $2.25, p=.01, d=.31$. As predicted by patterns in language (Study 1), the distinction between memory for individuals

\footnotetext{
${ }^{7}$ Results from Study 1 motivate directed predictions, and so one-tailed planned contrasts are reported.
} 
involved in intentional and accidental events was more pronounced for Spanish speakers $(M=4.25, S E=1.65)$ than for English speakers $(M=-80, S E=1.74), t(208)=$ $2.09, p=.02, d=.29$. This cross-linguistic difference was also consistent across events, $t(15)=2.02, p=.03$.

In addition to equivalent memory for agents of intentional events, English and Spanish speakers did not differ in their memory for the orientation of objects in the objectorientation memory task $(M=75.09, S E=1.42$ and $M=$ $73.53, S E=1.42$, respectively), $t(208)=.77, n . s$. This helps ensure that the cross-linguistic differences in eye-witness memory for accidents are not due to more general differences in memory capacity between the two groups. ${ }^{8}$

We note that our results do not show that Spanish speakers are unable to remember the individuals involved in accidental events, nor would such a finding be predicted from patterns in language. Of course, the overall level of performance on a memory task depends on many factors, including how many items there are to remember, the confusability of the items, and the length of the delay between encoding and test. As a result, any measure of memory performance can only be interpreted comparatively; it cannot be taken as a meaningful absolute index of memory ability. That is, getting $70 \%$ right will mean very different things depending on how hard the task is.

Rather than absolute levels of performance, the key finding here is a reliable difference in eye-witness memory between speakers of two different languages, specifically in the case of accidental events. The difference constitutes about $10 \%$ of the measurement range (from chance performance at $50 \%$ to a ceiling of $100 \%$ ) and is within the typical range of effect sizes in eye-witness memory research (see Shapiro \& Penrod, 1986, for a review).

\section{Discussion}

English and Spanish speakers remembered agents in a pattern consistent with event descriptions in their respective linguistic communities. Both groups described intentional events agentively and had similarly strong memory for the agents of these events. When it came to accidental events, however, English speakers used more agentive descriptions than did Spanish speakers and also remembered the agents of these events better than did Spanish speakers. It is not the case that Spanish speakers had poorer memory than English speakers more generally. The two groups showed similar memory for agents of intentional events as well as for object-orientation. Only accidental events were described and remembered differently across communities.

\footnotetext{
${ }^{8}$ Patterns revealed by analyses of memory for agents remain the same when object-orientation memory performance is included as a covariate.
}

These findings show a close coupling between the way events are typically talked about in a linguistic community and what people encode and remember about these events even when they are not talking.

Our results demonstrate that our eye-witness memories for even such momentary events as popping a balloon or breaking a pencil may be susceptible to influence from linguistic patterns that differ across natural languages. These results extend previous work on the role of language in eye-witness memory (e.g., Gentner \& Loftus, 1979; Loftus \& Palmer, 1974) to the cross-linguistic domain. In this case, typical ways of talking in one's linguistic community predict patterns in people's eye-witness memory for who did what. We should note that in important real-world domains like eye-witness memory, even a small difference in performance can have serious consequences: it could make the difference between a life behind bars or getting away with murder, between being falsely accused or exonerated.

Further, we find cross-linguistic differences in memory even though participants were not asked to describe the events at any time during the memory task. This example shows a deeper effect of language than what has been observed previously, for example in the case of manner of motion. It appears that an explicit requirement for linguistic description is not necessary to observe cross-linguistic differences in the case of causal events (see also Fausey, Long, Inamori \& Boroditsky, 2010 for a replication in Japanese, and Wolff, Jeon, \& Li, 2009). One possible reason for this difference is that the causal events included in our study are more complex than the motion events studied previously and as a result require more cognitive construal. For example, observers must integrate information about the basic physics of the event (e.g., whether the person touched the balloon, whether the balloon popped, whether he touched it right before it popped) with more social cues about the individual's state of knowledge and intentions (e.g., whether he meant to touch the balloon, whether he knew the balloon was there, whether he was surprised at the outcome). The need to integrate many different types of information to construe an event may leave some events especially susceptible to linguistic and cultural influences. Of course, many of the events and outcomes we observe and reason about in everyday life are far more complex than popping a balloon or breaking a pencil and may be even more strongly influenced by patterns in language.

What are the mechanisms by which language may modulate memory in the case of causal events? One possibility is that (even in situations when they are not required to describe) people spontaneously generate subvocal descriptions of events, and these descriptions encode specific details that happen to be useful in later memory 
tasks. For example, if one (subvocally) generated and stored a description like "The guy in the yellow shirt popped the balloon," this stored description could then be useful in later reporting whether it was the guy in yellow or in blue that popped the balloon. That is, such specific descriptions could serve as a secondary code, explicitly encoding information that would turn out to be relevant in a memory test. The way that English and Spanish speakers described events in Study 1 suggests that this mechanism is unlikely to be the source of the memory difference we observed between the two language groups. Very few descriptions (less than $3 \%$ in either language community) included the kind of identifying information that could help distinguish between the two actors. People most often referred to the actors as simply "a man" or "a guy" ("un hombre," "un joven" in Spanish), descriptions that are not specific enough to be diagnostic in distinguishing the two actors.

Another possibility is that rather than serving as a specific secondary code (e.g., by explicitly encoding testrelevant information like shirt color), language may modulate memory by directing people's visual attention as they witness events. For example, if one would most often hear (and produce) agentive descriptions like "He popped the balloon," this may orient visual attention to the agent and make one more likely to represent who that "he" is. There are (at least) two interesting possibilities for how language could shape attention in this way: an in-themoment effect or a more general effect. If people automatically generate internal linguistic descriptions of events (even in situations when they are not required to speak), it could be these internal descriptions that then bias people's attention in the moment. Alternatively, exposure to more or less agentive language in one's linguistic environment may create general attentional biases that do not require access to linguistic processes or linguistic descriptions in the course of the task. Speakers of languages that rely heavily on agentive language may become more likely to pay attention to and encode details about agents whether or not they generate any kind of internal linguistic description in the moment.

One direction for future studies would be to disrupt people's access to fluent linguistic processing during encoding (e.g., with verbal interference). Whether or not verbal interference affects memory outcomes may help us distinguish whether patterns in language shape attention in the moment (because linguistic descriptions are automatically generated and meddle in cognition even when people do not plan to speak) or by training general attentional biases. Both of these mechanisms would serve as interesting examples of how patterns in a linguistic environment can importantly shape what people encode and remember about the events they witness.

\section{Conclusions}

These findings suggest that our eye-witness memories for events may be influenced by the languages we speak. Speakers of different languages remember different things about the same events. Whether or not we are likely to remember who did what appears to pattern with how such events are normally described in our language community.

Acknowledgement We thank F. Leach, C. Lew-Williams, and D. Remus (acting talent), R. Bahamondes, G. Jenkins and M.P. Zúñiga (data collection), K. Moghbeli (programming), and M. Gutierrez and T. Shih (reliability coding). This research was funded by an NSF Graduate Research Fellowship to CMF and NSF Grant No. 0608514 .

\section{References}

Billman, D., \& Krych, M. (1998). Path and manner verbs in action: Effects of "skipping" or "exiting" on event memory. Proceedings of the 20th annual meeting of the Cognitive Science Society. Mahwah, NJ: Lawrence Erlbaum Associates.

Dorfman, A. (2004). Footnotes to a double life. In W. Lesser (Ed.), The genius of language (pp. 206-217). New York: Random House.

Fausey, C. M., \& Boroditsky, L. (2010). Subtle linguistic cues influence perceived blame and financial liability. Psychonomic Bulletin \& Review, 17(5), 644-650.

Fausey, C. M., Long, B. L., Inamori, A., \& Boroditsky, L. (2010). Constructing agency: the role of language. Frontiers in Cultural Psychology. doi:10.3389/fpsyg.2010.00162

Filipovic, L. (2007). Language as a witness: Insights from cognitive linguistics. The International Journal of Speech, Language and the Law, 14(2), 245-267.

Finkbeiner, M., Nicol, J., Greth, D., \& Nakamura, K. (2002). The role of language in memory for actions. Journal of Psycholinguistic Research, 31, 447-457.

Gennari, S. P., Sloman, S. A., Malt, B. C., \& Fitch, W. T. (2002). Motion events in language and cognition. Cognition, 83(1), 49-79.

Gentner, D., \& Loftus, E. F. (1979). Integration of verbal and visual information as evidenced by distortions in picture memory. American Journal of Psychology, 92(2), 363-375.

Grimshaw, J. (1982). On the lexical representation of romance reflexive clitics. In J. Bresnan (Ed.), Mental representations of grammatical relations. Cambridge: MIT Press.

Loftus, E. F., \& Palmer, J. C. (1974). Reconstruction of automobile destruction: An example of the interaction between language and memory. Journal of Verbal Learning and Verbal Behavior, 13, $585-589$.

Maldonado, R. (1992). Middle voice: The case of Spanish se. Dissertation Abstracts International, 53(07), 2351A. UMI no. 9235939.

Martinez, I. (2000). The effects of language on children's understanding of agency and causation. Dissertation Abstracts International, 61 (10), 3976A. UMI no. 9990936.

Oh, K. (2003). Language, cognition, and development: Motion events in English and Korean. Dissertation Abstracts International, 64 (09), 4655B. UMI no. 3105326.

Papafragou, A., Hulbert, J., \& Trueswell, J. (2008). Does language guide event perception? Evidence from eye movements. Cognition, 108, 155-184. 
Papafragou, A., Massey, C., \& Gleitman, L. (2002). Shake, rattle, 'n' roll: The representation of motion in language and cognition. Cognition, 84(2), 189-219.

Shapiro, P. N., \& Penrod, S. (1986). Meta-analysis of facial identification studies. Psychological Bulletin, 100, 139-156.

Slobin, D. I. (2003). Language and thought online: Cognitive consequences of linguistic relativity. In D. Gentner \& S. GoldinMeadow (Eds.), Language in mind: Advances in the study of language and thought (pp. 157-191). Cambridge, MA: MIT Press.
Slobin, D. I., \& Bocaz, A. (1988). Learning to talk about movement through time and space: The development of narrative abilities in Spanish and English. Lenguas Modernas, 15, 5-24.

Trueswell, J. C., \& Papafragou, A. (2010). Perceiving and remembering events cross-linguistically: Evidence from dual-task paradigms. Journal of Memory and Language, 63(1), 64-82.

Wolff, P., Jeon, G., \& Li, Y. (2009). Causers in English, Korean and Chinese and the individuation of events. Language and Cognition, 1(2). 